\section{In the film business}

\section{Andrew Holmes-Siedle}

The SI-SiO, System. Edited by Pieter Balk. Elsevier: 1988. Pp. 356. Dfl. 220, $\$ 115.75$.

The Physics and Chemistry of $\mathrm{SIO}_{2}$ and the SI-SIO ${ }_{2}$ Interface. Edited by C. Robert Helms and Bruce E. Deal. Plenum: 1988. Pp.556. \$138, £86.25.

ONE reason for the low cost of massproduced microelectronics is the abundance of silicon on the Earth. Another reason, not so widely recognized, is the versatile nature of the oxide of silicon, silica $\left(\mathrm{SiO}_{2}\right)$

When silicon is heated in oxygen or steam, a strong, tenacious form of silica grows as continuous film. This oxide film is a very useful working material for fabricating microelectronic structures. First and most important, the films are excellent insulators and are used as the oxide in the metal-oxide-semiconductor sandwich which is used widely in microelectronics. Second, the films can be patterned by photolithographic etching; this is what gives us the ability to make very fine patterns of $p-n$ junctions by diffusion.

In both applications, the classical view was that silica was an inert spacer or barrier, unaltered by the application of fields or other stresses. The reality is different, and not surprising once we realize what a sensitive environment the silica has around it. Even tiny electronic changes in the silica are picked up in the sensitive layer of silicon underneath it and are reflected in the performance of the electronic device concerned. If the film fails, the device dies. Hence the reason why a large number of researchers are trying to understand the growth of silica films, their structure, and the electronic states which occur within the films and (especially important) at the interface between the film and the silicon wafer beneath.

Pieter Balk is a European authority on the silica film and in his book he has brought together a coherent set of contributions on the subject. By contrast, the volume edited by Helms and Deal is a snapshot of research in 1988 . It contains over 60 short papers on $\mathrm{SiO}_{2}$ and its interfaces that were delivered at a conference held in Atlanta in May 1988. In the classical view, insulators such as silica were not meant to carry currents at all unless total breakdown occurred. Contemporary research is defining how, in fact, charge enters, passes through and is trapped in the oxide film. An especially active region is the $\mathrm{Si}-\mathrm{SiO}_{2}$ interface, where charge- trapping states have special ability to exchange electrons rapidly with the silicon. The standard of science required to unravel this picture is extremely high, despite the 'applied' nature of the problem.

The contributors to Helms and Deal present useful vignettes of the latest research but, despite the inclusion of some review papers, there is not much in the way of a coherent view of the subject. Some of the research papers are outstanding, however - for example those by Irene on the growth of oxides on silicon at high temperatures, by Bevk on the flatness or otherwise of the interface between single crystal $\mathrm{Si}$ and grown $\mathrm{SiO}_{2}$ on the atomic scale, and by the late Frank Feigl and his school on charges in $\mathrm{SiO}_{2}$. Damage to the silica film due to charge injection or irradiation is a very important subject and papers on this topic occupy a whole section of the book. Different but equally fascinating approaches to damage effects are taken by Edwards, Schulz and Ma. No less important are a contribution by Deal, which attempts to define what constitutes 'quality' in this use of silica, and one by Di Maria on charge transport in silica.

There can be few better people to organize a summary of insulator films than Balk. He himself contributes a long and masterly introductory section to The

\section{Off target}

\section{Tony Davies}

Antibody-Mediated Dellvery Systems. Edited by John D. Rodwell. Marcel Dekker: 1988. Pp. 384. \$99.75.

HYBRIDOMAS growing in vitro or in vivo can generate large quantities of antibody molecules with a wide range of binding specificities. That capacity has acted as a catalyst to the concept that drugs can be taken to their targets on the back of carrier molecules. The broad strategies are now well known in that an antibody molecule, that can specifically bind to a target such as a tumour, is covalently linked to another and cytotoxic agent. Such heterobifunctional conjugates, it is argued, will kill their targets without serious nonspecific side effects.

The present volume is a collection of papers describing these possibilities. There is no editorial summary, nor apparently has any serious attempt been made to avoid repetition at the beginning of each chapter. On the positive side the accounts of materials and methods are relegated to a technical appendix in each paper, thus preserving the notion that review is intended. In fact the contributions are in most instances fairly standard
$\mathrm{Si}_{-} \mathrm{SiO}_{2}$ System. Four chapters are written by European authors, who are not only of high scientific standing but also explain themselves very clearly. Another is by Robert Helms, an editor of the other book reviewed here. Although many of the authors have spent long periods working in the United States, Balk's book demonstrates Europe's strength in the thin-film dielectrics field - a fortunate state of affairs considering the need for this skill in a competitive industry. The practical aspects of the use of silica films in microelectronics are nicely integrated with the explanations of the complex physics of the system.

In an apparently off-hand comment, Balk says that "The prescription for optimising MOS properties is basically a very straightforward one. The $\mathrm{SiO}_{2}$ should be as perfect a network as possible...". This remark in fact encompasses neatly the whole complex and vital activity which forms the topic of these books. How well this prescription is met may make the difference between success and failure in one of the most crucial industries of the twentieth and (perhaps) the twenty-first centuries.

Andrew Holmes-Siedle is owner of REM Instruments, 64A Acre End Street, Eynsham, Oxford OX8 1PD, UK, and a consultant to the Fulmer Research Laboratories, Stoke Poges, near Slough.

and presumably unrefereed research papers, which mainly portray the interests of their authors rather than attempting to appraise a particular aspect of the field.

Various conjugates of conventional chemotherapeutic agents with antibodies are considered, but although the experimental results are promising - as they always have been - the extension into clinical practice seems to be slow to mature. Some of the more esoteric approaches using, for example, cobra venom factor are portrayed - at least those tried in vitro. Frankel presents a useful review of linkage of proteinaceous toxins to antibodies. There is even a paper (for no obviously good reason) on methods of labelling with ${ }^{67} \mathrm{Cu}$ using porphyrins.

By picking and choosing, it is possible to find in this volume the states of various branches of the targeting art, but it is rather hard work. If, as is threatened, this is to be the first of a series of books on roughly the same topic, much more care should be given to selection and editing of papers so that the standards of critical review and redaction that readers have every right to expect are fulfilled.

Tony Davies is Deputy Director of the Institute of Cancer Research, Haddow Laboratories, 15 Cotswold Road, Belmont, Sutton, Surrey SM2 5NG, UK. 